\title{
Inferring connection proximity in networks of electrically coupled cells by subthreshold frequency response analysis
}

\author{
Corrado Calì • Thomas K. Berger • Michele Pignatelli • \\ Alan Carleton • Henry Markram • Michele Giugliano
}

Received: 14 May 2007 /Revised: 2 October 2007 / Accepted: 8 October 2007 / Published online: 28 November 2007

(C) Springer Science + Business Media, LLC 2007

\begin{abstract}
Electrical synapses continuously transfer signals bi-directionally from one cell to another, directly or indirectly via intermediate cells. Electrical synapses are common in many brain structures such as the inferior olive, the subcoeruleus nucleus and the neocortex, between neurons and between glial cells. In the cortex, interneurons have been shown to be electrically coupled and proposed to participate in large, continuous cortical syncytia, as opposed to smaller spatial domains of electrically coupled cells. However, to explore the significance of these findings it is imperative to map the electrical synaptic microcircuits, in analogy with in vitro studies on monosynaptic and disynaptic chemical coupling. Since "walking" from cell to cell over large distances with a glass pipette is challenging, microinjection of (fluorescent) dyes diffusing through gap-
\end{abstract}

Action Editor: Xiao-Jing Wang

Corrado Calì and Thomas K. Berger are equally contributing authors.

C. Calì · T. K. Berger • M. Pignatelli •

H. Markram • M. Giugliano

Laboratory of Neural Microcircuitry, Brain Mind Institute,

École Polytechnique Fédérale de Lausanne (EPFL),

1015 Lausanne, Switzerland

C. Calì

Department of Electronics, Polytechnic of Turin,

10129 Turin, Italy

M. Pignatelli $\cdot$ A. Carleton

Flavour Perception Group, Brain Mind Institute, École Polytechnique Fédérale de Lausanne (EPFL),

1015 Lausanne, Switzerland

M. Giugliano $(\bowtie)$

EPFL SV BMI LNMC, Station 15,

1015 Lausanne, Switzerland

e-mail: michele.giugliano@epfl.ch junctions remains so far the only method available to decipher such microcircuits even though technical limitations exist. Based on circuit theory, we derive analytical descriptions of the AC electrical coupling in networks of isopotential cells. We then suggest an operative electrophysiological protocol to distinguish between direct electrical connections and connections involving one or more intermediate cells. This method allows inferring the number of intermediate cells, generalizing the conventional coupling coefficient, which provides limited information. We validate our method through computer simulations, theoretical and numerical methods and electrophysiological paired recordings.

Keywords Gap-junctions · Electrical coupling · Networks · ZAP current $\cdot$ Impedance $\cdot$ Interneurons $\cdot$ Cortex $\cdot$ Layer VI

\section{Introduction}

As opposed to chemical synaptic transmission, electrical coupling mediated by gap-junctions constitute a "communication channel" in which information is conveyed continuously and bi-directionally. Although they provide direct pathways supporting electrical - as well as biochemical - signalling between contacting cells, their role in shaping neuronal activity has been underestimated until recently (Hormuzdi et al. 2004). Today, our knowledge about the functional role of electrical synapses considerably increased through the development of a variety of new methods (Bennett and Zukin 2004).

In the cortex, gap-junctions have been implicated in the synchronization of gamma oscillations (Traub et al. 2001) and it has been found that neocortical GABAergic interneurons establish non-random electrical networks 
(Hestrin and Galarreta 2005), whose spatial extension might be unexpectedly wide (Amitai et al. 2002). A complete characterization of the electrically coupled microcircuits is technically challenging but it appears imperative to further advance our understanding of the role of gapjunction in emerging network activities (Beierlein et al. 2000; Gao and Holmes 2006; Ermentrout et al. 2004; Lewis and Rinzel 2003; Traub 1995).

In analogy to the impact that in vitro paired-recordings and infrared differential-interference-contrast microscopy had on the study of the synaptic organization of the brain, recent multi-patching techniques will certainly be beneficial to the study of electrical neuronal networks. In fact, it is becoming increasingly common for electrophysiologists to access rigs equipped with 2 to 12 micromanipulators, and the feasibility and efficiency of brain slice studies, focusing on the (patho-) physiology and plasticity of (chemical) synaptic transmission, showed great improvements (Le Be and Markram 2006). Not only direct or monosynaptic connections between neurons could be characterized in depth, but also the stereotypical features of indirect or polysynaptic connections could be explored (Silberberg and Markram 2007).

However, while studies on the (chemical) synaptic organization are becoming increasingly accurate and detailed, our understanding of the electrical coupling in the cortex as well as in other brain region is still incomplete. The systematic direct optical observation of electrical networks of neurons or of glial cells, employing diffusible (fluorescent) tracers, was not versatile enough so far (but see Hoshi et al. 2006; Dupont et al. 2006) and it had serious limitations as the dyes do not always diffuse from one cell to another despite the electrical coupling. In addition, no obvious electrophysiological protocol is available either to discriminate between "monosynaptic" or "polysynaptic" electrical coupling [Fig. 1(a-b)] or to infer the connection proximity (i.e. the number of intermediate cells). The conventional coupling coefficient, for instance, quantifies the ratio of membrane voltage deflections induced in two connected neurons upon current injection in one of them, but it is only a qualitative characterization of the coupling (a)

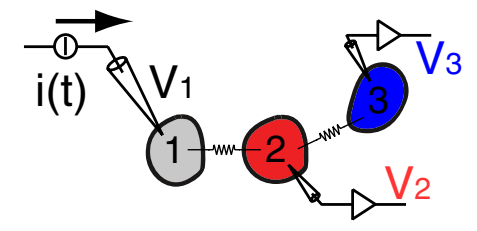

(c)

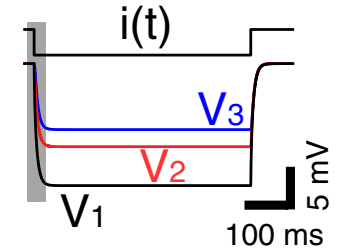

(f)

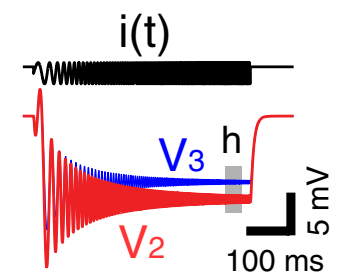

(d)

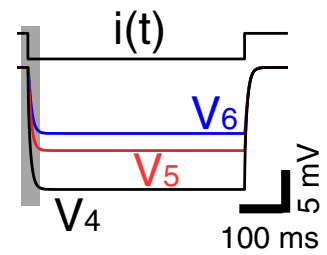

(g)

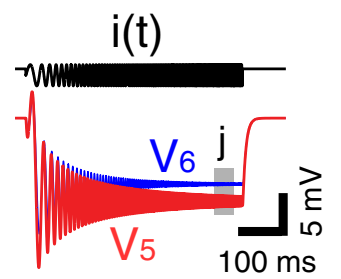

(b)

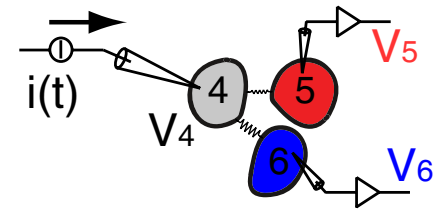

(e)

$\mathrm{i}(\mathrm{t})$

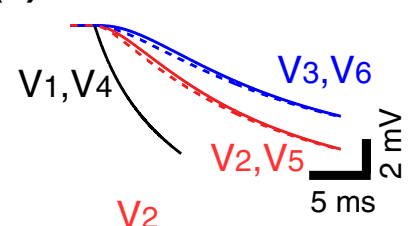

(h)

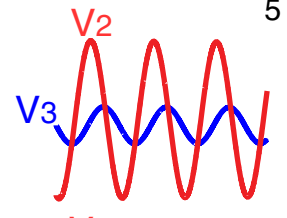

(j)

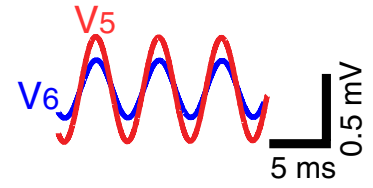

Fig. 1 Probing electrical coupling between neurons by AC and DC protocols. Sample model networks of three conductance-based pointneurons $(\mathbf{a}-\mathbf{b})$ are made identical, except for the topology and the strength of electrical coupling. Assessing the coupling coefficients (c.c.) requires hyperpolarizing one cell (c-d) and computing the ratio between evoked voltages at the steady-state. For the sake of illustration, junctional conductances were tuned to result in (large) identical c.c., equals to $\mathrm{V}_{2} / \mathrm{V}_{1}=\mathrm{V}_{5} / \mathrm{V}_{4}=0.94$ and $\mathrm{V}_{3} / \mathrm{V}_{1}=\mathrm{V}_{6} / \mathrm{V}_{4}=0.91$. Discriminating between (a) and (b) is therefore not possible (c-d), although responses slightly differ during the first $5 \mathrm{~ms}$ (e). Upon ZAP-currents injection (f-g), (a) and (b) can be now easily discriminated: high-frequency attenuations and phase shifts $(\mathbf{h}-\mathbf{j})$ clearly differ (offsets graphically subtracted). Simulation parameters: cells capacitance and input resistances $\mathrm{C}=132.7 \mathrm{pF}, \mathrm{R}_{1}=\mathrm{R}_{4}=121.2 \mathrm{M} \Omega$, $\mathrm{R}_{2}=\mathrm{R}_{5}=95.1 \mathrm{M} \Omega$, and $\mathrm{R}_{3}=\mathrm{R}_{6}=96.5 \mathrm{M} \Omega$; coupling strengths $\mathrm{R}_{12}=$ $\mathrm{R}_{23}=25, \mathrm{R}_{46}=22.43, \mathrm{R}_{45}=12.2 \mathrm{M} \Omega ; \mathrm{DC}$ current-step amplitude $-300 \mathrm{pA}$; ZAP-current offset (graphically subtracted), amplitude and frequency range $-300 \mathrm{pA}, 500 \mathrm{pA}$ and $10-200 \mathrm{~Hz}$, respectively 
strength (Bennett and Zukin 2004) and it does not discriminate between weak direct connections and indirect connections [i.e. mediated by intermediate cells-see Fig. 1(c-d)].

In this work, we introduce an analytical formalism for describing the subthreshold AC coupling in large networks of electrically coupled isopotential cells. This theoretical framework leads us to suggest an electrophysiological protocol to infer the proximity of coupling in networks of electrically coupled cells, complementing existing approaches. This method is similar to the subthreshold membrane impedance characterization (Hutcheon and Yarom 2000) but focuses on the transfer-impedance across cells. By intracellular linear swept sine waves (ZAP) current injections in one cell and Fourier analysis of the subthreshold responses in electrically coupled cells, we prove that it is possible to infer how many intermediate cells are between the selected pair.

We verify this method analytically as well as numerically by means of computer simulations of conductancebased point neurons as well as in networks of simple multicompartmental neurons, pointing out its assumptions and limitations.

\section{Methods}

2.1 Neuronal modeling and symbolic electrical network analysis

Computer simulations were performed using NEURON (Carnevale and Hines 2006) to verify our analytical and numerical results. Small networks of single- and multicompartmental neurons were characterized by reduced geometries and simplified topologies. Parameters were generated randomly to obtain heterogeneous networks (i.e. membrane time-constants in the range $[10 ; 25] \mathrm{ms}$; input-resistances in the range $[75 ; 190] \mathrm{M} \Omega$ ). Neurons incorporated leak and voltage-dependent ion currents, such as sodium and potassium conductances. Non-rectifying voltage-independent electrical gap-junctions were defined and simulated following Migliore et al. (2005). For the sake of illustration, the resistance of electrical coupling was chosen arbitrarily, in order to result in strong coupling coefficients and good signal-to-noise ratio for our analysis. Membrane voltage outputs from simulations were stored on disk and processed off-line in MATLAB (The Mathworks, Natick, MA, USA).

In the case of (passive) single-compartmental model networks, computer-assisted symbolic electrical network analysis (Gielen et al. 1994) was systematically employed to compute transfer-impedances analytically. Several 1-dimensional and 2-dimensional symmetric and asymmetric networks composed of a finite number of neurons were then considered to test our thesis.

Simulation code and analysis scripts are available from ModelDB http://senselab.med.yale.edu/modeldb via accession number 94321.

\subsection{Transfer-impedance}

In the definition of our protocol, we considered linear swept sine waves (ZAP) for current injection, as described for exploring frequency responses in single (model) neurons (see Hutcheon and Yarom 2000). Such waveforms take the form [Figs. 1(f-g), 2]

$$
\begin{aligned}
I_{Z A P}(t) & =I_{0}+A \cdot \sin \left(2 \cdot \pi \cdot \int_{0}^{t} f(\xi) \cdot d \xi\right) \\
& =I_{0}+A \cdot \sin \left(2 \cdot \pi \cdot \frac{f(t)+f_{0}}{2} \cdot t\right) \quad 0 \leq t \leq t_{1},
\end{aligned}
$$

where the instantaneous frequency $f(t)$ linearly sweeps from $f_{0}$ to $f_{1}$, in $\left[0 ; t_{1}\right]$ :

$f(t)=f_{0}+\left(f_{1}-f_{0}\right) \cdot t / t_{1} \quad 0 \leq t \leq t_{1}$.

Typical values for $I_{0}, A, f_{0}, f_{1}, t_{1}$ were $-400-0 \mathrm{pA}$, $100-500 \mathrm{pA}, 10 \mathrm{~Hz}, 200-1,000 \mathrm{~Hz}$, and 1-30 s, respectively.

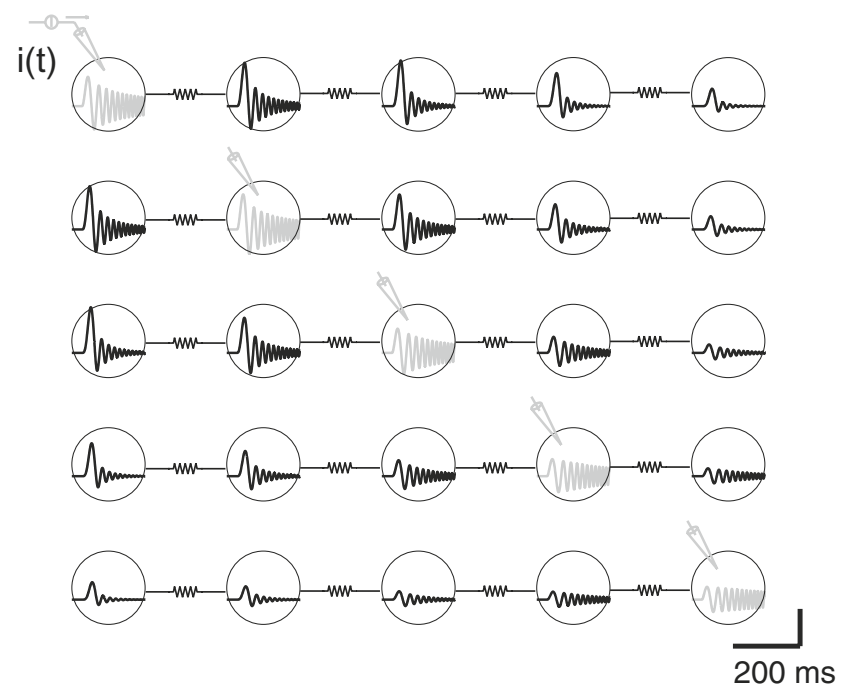

Fig. 2 Computer simulations of a "chain" of 5 heterogeneous conductance-based model neurons. Simulations were repeated 5 times, injecting identical ZAP currents $i(t)$ into each cell, one at a time. Voltage transients simultaneously evoked in all the cells are plotted in black, subtracting individual offsets and under exponential rescaling (i.e. vertical calibration was chosen as $210^{-\mathrm{d}} \mathrm{mV}$, with " $\mathrm{d}$ " the distance between the recorded cell - black - and the injected cell gray $-\mathrm{d}=1, \ldots, 4)$. Note that reciprocity is not necessarily preserved as a consequence of the heterogeneity in cells input resistances. Simulation parameters: cells capacitance $\mathrm{C}=132.7 \mathrm{pF}$ and input resistances randomly selected in the range 70-180 $\mathrm{M} \Omega$; coupling strengths $\mathrm{R}_{\text {gap }}=25 \mathrm{M} \Omega$; DC current-step amplitude $-300 \mathrm{pA}$; ZAPcurrent offset, amplitude and frequency range $-300 \mathrm{pA}, 500 \mathrm{pA}$ and 10-200 Hz, respectively 
In addition, with the aim of improving the signal-to-noise ratio in real electrophysiological experiments, the amplitude $A$ could be also chosen as an increasing function of time, such as

$A(t)=A_{0}+\left(A_{1}-A_{0}\right) \cdot t / t_{1}$.

A similar approach has previously been used to investigate somatic and dendritic membrane frequency resonances (Hutcheon and Yarom 2000; Ulrich 2002). In those cases, the membrane impedance $(\mathrm{Z})$ at a given holding potential was evaluated as the ratio between Fourier transforms of the voltage response and the current input [Eq. (1)].

Here, we address the transfer-impedance $\left(\mathrm{Z}_{\mathrm{ab}}\right)$ between two electrically coupled cells (e.g. cells 'a' and 'b'), computed as the ratio of Fourier transforms of the voltage responses $\left(\mathrm{V}_{\mathrm{a}}\right.$ and $\left.\mathrm{V}_{\mathrm{b}}\right)$ recorded simultaneously, upon current injection $\mathrm{I}_{\mathrm{ZAP}}$ in one of the two cells. As opposed to the analysis of $\mathrm{Z}$, we restrict our interest to the analysis of the magnitude and the phase shift of $Z_{a b}$ mainly at high frequencies (i.e. above $100 \mathrm{~Hz}$ ), neglecting the contributions of subthreshold resonances. In a real experiment, one could best approximate such conditions, by applying intraand extracellular pharmacological blockers of intrinsic voltage-dependent membrane currents.

\subsection{Phenomenological modeling of transfer-impedances}

Electrically coupled isopotential cells can be considered identical to electrical networks of R-C circuit elements, at least in the high-frequency subthreshold regime. For such dynamical systems, a linear differential relationship relating any two membrane potentials $\mathrm{V}_{\mathrm{a}}$ and $\mathrm{V}_{\mathrm{b}}$ exists (Horowitz and Hill 1989; Brogan 1991):

$$
\begin{aligned}
a_{n} & \cdot d^{n} V_{a} / d t^{n}+\ldots+a_{1} \cdot d V_{a} / d t+a_{0} \cdot V_{a} \\
& =b_{m} \cdot d^{m} V_{b} / d t^{m}+\ldots+b_{1} \cdot d V_{b} / d t+b_{0} \cdot V_{b},
\end{aligned}
$$

where $V_{a}$ and $V_{b}$ are referred to a subthreshold holding membrane potential and no external current injection has been included.

In the frequency-domain, Eq. (4) is equivalent to the algebraic relationship $\mathrm{W}_{\mathrm{a}}=\mathrm{Z}_{\mathrm{ab}}(\mathrm{j} \omega) \mathrm{W}_{\mathrm{b}}$, where $j=\sqrt{-1}$, $\omega=2 \pi \mathrm{f}$ is the angular frequency, $\mathrm{W}_{\mathrm{a}}$ and $\mathrm{W}_{\mathrm{b}}$ are the Fourier transforms of $V_{a}$ and $V_{b}$, respectively. $Z_{a} b$ is called transfer-impedance between $V_{a}$ and $V_{b}$, taking the form of a rational complex function [e.g. see Eqs. (14) and (15)]

$$
\begin{aligned}
Z_{a b}(j \cdot \omega)=G_{0} & \cdot\left[\frac{\left(j \cdot \omega+z_{1}\right) \cdot\left(j \cdot \omega+z_{2}\right) \cdot \ldots \cdot\left(j \cdot \omega+z_{m}\right)}{\left(j \cdot \omega+p_{1}\right) \cdot\left(j \cdot \omega+p_{2}\right) \cdot \ldots \cdot\left(j \cdot \omega+p_{n}\right)}\right] \\
& \cdot\left[\frac{p_{1} \cdot p_{2} \cdot \ldots \cdot p_{n}}{z_{1} \cdot z_{2} \cdot \ldots \cdot z_{m}}\right] .
\end{aligned}
$$

$G_{0}$ represents the DC-coupling attenuation (i.e. $\omega=0$ ) and it coincides with the coupling coefficient. $z_{i}$ and $p_{i}$ are in general complex numbers, referred to as transmission zeroes and poles. We recall that $\left\{p_{i}\right\}$ are eigenvalues of Eq. (4) and that $\left\{z_{i}\right\}$ and $\left\{p_{i}\right\}$ are roots of the polynomials with coefficients $\mathrm{b}_{m}, \ldots, \mathrm{b}_{0}$ and $\mathrm{a}_{n}, \ldots, \mathrm{a}_{0}$, respectively. The value of $\mathrm{G}_{0}$, the number and values of zeroes and poles fully determine the profile of transfer-impedance, as often represented through logarithmic Bode diagrams (Brogan 1991) (Figs. 3 and 6). We note that at high-frequency ( $\omega>$ $\left.\max \left\{z_{i}, p_{i}\right\}\right) \mathrm{Z}_{\mathrm{a} \mathrm{b}} \sim(\mathrm{j} \omega)^{m-n}$, so that its magnitude follows an integer power-law and the phase-shift is a multiple of $90^{\circ}$.

\subsection{Experimental preparation and electrophysiological recordings}

For the experiments reported in Fig. 8, neocortical parasaggital acute slices $(300 \mu \mathrm{m}$ thick) of the somatosensory cortex were prepared as described in (Silberberg and Markram 2007), from 15-days-old Wistar rats, in agreement with institutional guidelines and with the Swiss cantonal regulations for animal experimentation (license 1550.1, granted by the Veterinary Service of Canton Vaud, Lausanne). Layer 6 interneurons were visualized by differential interference contrast microscopy, routinely filled with biocytin and stained (Hsu et al. 1981). Multiple whole-cell patch-clamp recordings were made at $35^{\circ} \mathrm{C}$ from the soma of up to 6 cells with Axopatch 200B amplifiers under bridge-balanced current-clamp mode. Estimates of the membrane input resistance and time constant were $67.3 \pm 46.1 \mathrm{M} \Omega$ and $9.7 \pm$ $4.6 \mathrm{~ms}$ (mean $\pm \mathrm{std}, n=10$ ), respectively. The extracellular solution contained (in $\mathrm{mM}$ ) $125 \mathrm{NaCl}, 25 \mathrm{NaHCO}_{3}$, $2.5 \mathrm{KCl}, 1.25 \mathrm{NaH}_{2} \mathrm{PO}_{4}, 2 \mathrm{CaCl}_{2}, 1 \mathrm{MgCl}_{2}, 25$ glucose, bubbled with $95 \% \mathrm{O}_{2}, 5 \% \mathrm{CO}_{2}$. The intracellular solution contained (in $\mathrm{mM}$ ) $110 \mathrm{~K}$-gluconate, $10 \mathrm{KCl}, 10$ HEPES, 4 ATP-Mg, $0.3 \mathrm{Na}_{2}$-GTP, $10 \mathrm{Na}_{2}$-Phosphocreatine, as well as $0.3-0.4 \%$ Biocytin. The $\mathrm{pH}$ was adjusted to 7.3 with $\mathrm{KOH}$.

\section{Results}

\subsection{High-frequency properties of transfer-impedances $Z_{a b}$}

Let's consider a network of electrically coupled cells, each of them electrotonically compact. We ignore active biophysical properties, whose contribution is expected to be negligible for subthreshold high-frequency regimes. This leads to modeling each cell as a single R-C compartment.

Our main result is that the transfer-impedance between the subthreshold membrane voltages of two coupled cells is dominated by the (discrete) number $d$ of groups present 


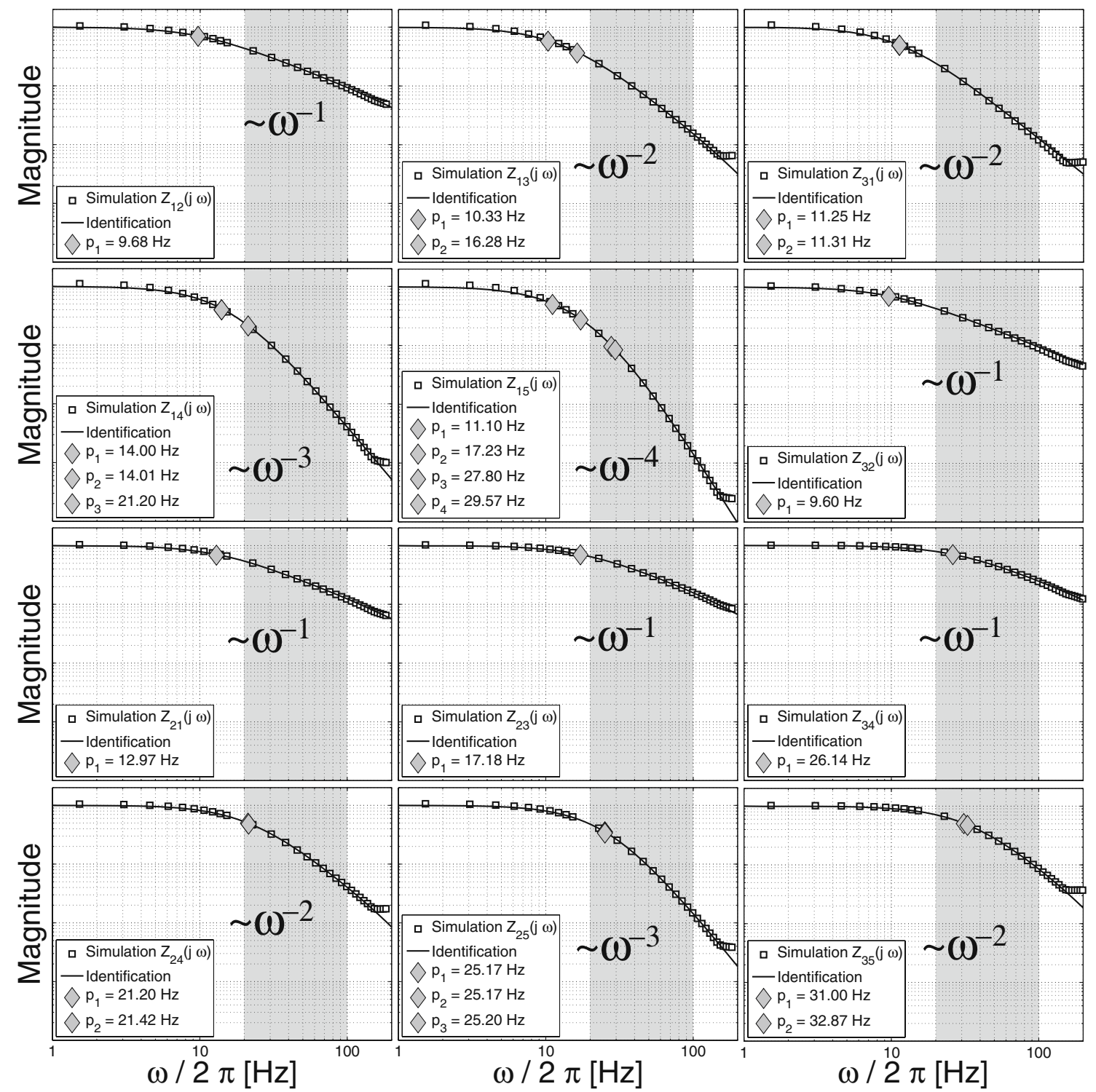

Fig. 3 The proximity of electrical coupling is inferred from the slope of the transfer-impedance in double logarithmic plots. Transferimpedances $Z_{a b}$ were computed as the ratios between the Fourier transforms of the voltage transients of Fig. 2 (i.e. "a" representing the index of the injected cell) (open squares). Proximity $|\mathrm{a}-\mathrm{b}|$, is operatively inferred from the number of numerically identified eigenvalues $\mathrm{p}_{\mathrm{i}}$ (filled diamonds) needed to fit $\mathrm{Z}_{\mathrm{ab}}$ (continuous line),

or equivalently by the integer slope of $Z_{a b}$ in the frequency range 20-100 Hz marked in gray. Simulation data $Z_{a b}$ have been all normalized to unitary gain (i.e. to the same coupling coefficient) and the steep change in slope above $200 \mathrm{~Hz}$ is caused by the limited upper frequency of the ZAP stimulus, employed in these simulations (i.e. linearly increasing from 10 to $200 \mathrm{~Hz}$ ). Simulation parameters as in Fig. 2

"in cascade" between the two cells. More specifically, the smallest number of intermediate cells that connect electrically two neurons coincides with the high-frequency powerlaw exponent of the transfer-impedance and it relates to its asymptotic phase-shift (Figs. 1(f-j) and 3): $Z_{a b} \sim(j \omega)^{-d}$.

Intuitively, the "series" of groups of capacitors and resistors result in the product of individual impedances, whose high-frequency behavior can be approximated as $(j \omega)^{-1}$. Then, the resulting power-law exponent is given by the sum of each exponent [e.g. $(j \omega)^{-1}(j \omega)^{-1}$ $(\mathrm{j} \omega)^{-1}=(\mathrm{j} \omega)^{-3}$ ].
This result is general and was verified in several network architectures by analytical and/or numerical methods. Furthermore, it is independent of the topologies tested, such as 1-dimensional finite chains with nearest(Figs. 2 and 3) and next-nearest-neighbor (not shown) electrical connectivity, 1-dimensional infinite (not shown) chains with nearest-neighbor connectivity, as well as 2-dimensional finite (not shown) and infinite networks (Fig. 6) with nearest-neighbor connectivity. In addition, this result was tested in finite asymmetric 1-dimensional network topologies (not shown) where some neurons had 
a heterogeneous number of neighbors (i.e. non-uniform non-symmetric loads).

The fact that just the shortest-path distance emerges in this context can be intuitively appreciated invoking the concept of the "parallel" of transfer-impedances. From system theory, a parallel of dynamical linear system is described as the sum of individual transfer functions. Then the term characterized by the weakest attenuation (i.e. the lowest power-law exponent-the shortest path) numerically prevails, because at high-frequency it is infinitesimal of a lower order [i.e. $(\mathrm{j} \omega)^{-1}+(\mathrm{j} \omega)^{-3} \sim(\mathrm{j} \omega)^{-1}$ ].

\subsection{Estimating connection proximity}

On the basis of these observations, a way exists for operatively estimating the connection proximity $d$. The strategy is schematically presented in Figs. 1 and 2, in which a comparison with the evaluation of the coupling coefficient is presented in an ambiguous situation. Given simultaneous access to the subthreshold membrane potential of $k$ cells by patch pipettes, an ideal experiment should proceed by injecting a ZAP current in one cell at a time, while recording the evoked voltage responses in all the cells. In order to prevent interpretative ambiguities related to zero-pole cancellations (see the next section), only the transfer-impedances between the membrane voltage $V_{m}(t)$ of the stimulated cell and those of all the other cells $V_{k}(t)$ should be evaluated. This is carried out by Fast-Fourier transforming voltage traces into $\mathrm{W}_{\mathrm{m}}(\mathrm{j} \omega)$ and $\mathrm{W}_{\mathrm{k}}(\mathrm{j} \omega)$ and subsequently taking their ratio $Z_{k m}=W_{k}(j \omega) / W_{m}(j \omega)$. Figure 2 reports graphically the proposed procedure for a chain of 5 conductance-based neurons electrically coupled to their nearest-neighbors.

Figure 3 reports the analysis of the network of Fig. 2, testing the validity of our method by means of numerical simulations performed in NEURON. For symmetry reasons, Fig. 3 includes only a subset of the transfer impedances $Z_{\mathrm{km}}(\mathrm{m}=1 . .3 ; \mathrm{k}=1 . .5, \mathrm{k} \neq \mathrm{m})$ obtained from simulated voltage recordings. Equivalent to linear regression in the double-logarithmic plane, we routinely fit to the results of the simulations (black squares) a reduced version of $Z_{\mathrm{km}}$ [i.e. with unitary numerator - see Eqs. (14) and (15)] (continuous line) by means of simulated annealing techniques (Press et al. 1992), extracting only the number of eigenvalues $p_{i}^{\prime}<500-1,000 \mathrm{~Hz}$ (diamonds). In fact, the optimization algorithm would move away those poles $p_{i}$ ' unnecessary for the fit, providing us with an estimate of $d$.

For instance, the analysis of the transfer-impedance between cell 1 and cell 5 was labelled as $Z_{1} 5$ in the legend, referring to the ZAP current injection in cell 1 and to the simultaneous recording of subthreshold membrane voltages in cells 5 and 1 . The proximity of electrical connection is $d=5-1$, which precisely coincides with the slope of the power-law impedance indicated as " $\sim \omega^{-4 "}$. It is interesting to note that the identified poles were relatively concentrated at low frequencies $\sim[10 ; 30] \mathrm{Hz}$.

\section{1-Dimensional networks of electrically coupled neurons as ladder networks}

In this section we study analytically a finite heterogeneous 1-dimensional network of passive isopotential neurons, electrically coupled by nearest-neighbor gap-junctions of identical conductance (Fig. 4). We demonstrate that the connection proximity can be inferred from the highfrequency properties of the transfer impedance between any two cells of the network. Such results have been verified through extensive NEURON computer simulations employing single-compartmental conductance-based spiking neurons (as in Fig. 3), through semi-symbolic crosscheck of the analytical expressions presented below, as well as by means of SSPICE, a symbolic electrical networks analysis programs for linear circuits (Wierzba et al. 1989; Gielen et al. 1994).

Let's consider an open "chain" of $n$ single-compartmental model neurons. Each neuron is coupled electrically to its two neighbors by voltage-independent gap-junctions of resistance $R_{\text {gap }}$, as sketched in Fig. 4(a). The impact of voltage-dependent currents to the subthreshold intrinsic transfer properties is assumed to be negligible in the high

(a)

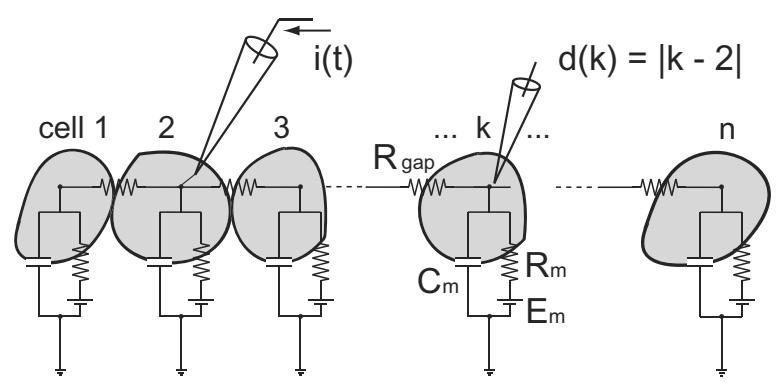

(b)

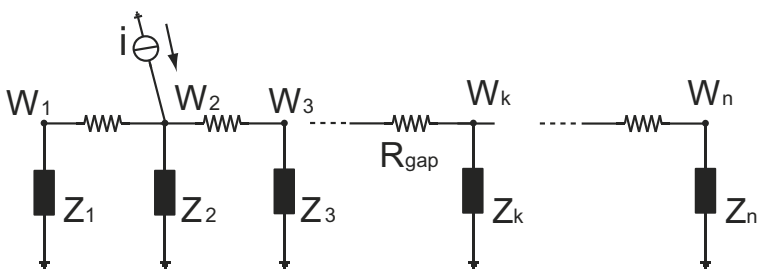

Fig. 4 From electrical equivalents to transfer impedances in 1-dimensional networks. The (subthreshold) electrical behavior of single-compartmental model neurons was represented by their passive membrane properties (a). The connection proximity between neurons can be inferred by the transfer impedance properties between the site of current injection and any other circuit node. This is a consequence of the discrete number of groups R-C on the shortest path between the two cells. In (b), the equivalent ladder network has been sketched to guide the analytical derivation of the cell-to-cell iterative transfer impedance [see Eqs. (9-12)] 
frequency band (i.e. $>100 \mathrm{~Hz}$ ). Each cell can be therefore described by a RC circuit (Weiss 1996) and the resulting equivalent electrical circuit [Fig. 4(b)] can be studied by standard techniques. We indicate the membrane voltage of the $k$-th cell as $V_{k}(t)$ referred to the resting potential (i.e. $\left.E_{m k}\right)$, and we evaluate its Fourier transform $W_{k}(\omega)$ from the definition

$$
W_{k}(j \cdot \omega)=\int_{-\infty}^{+\infty} V_{k}(t) \cdot e^{-j \cdot \omega \cdot t} \cdot d t
$$

where $j=\sqrt{-1}$ and $\omega=2 \pi \mathrm{f}$ is the angular frequency. By this transformation, any input-output differential relationships governing the circuit of Fig. 4 become algebraic (Horowitz and Hill 1989).

The circuit of Fig. 4 is a ladder network composed of linear bipolar elements (Feynman 1964). It is now possible to derive iterative mathematical expressions that relates $\left\{W_{k}(\omega)\right\}$ to each other and to a set of externally injected currents $\left\{I_{k}\right\}$ :

$$
\left\{\begin{array}{c}
W_{1} / Z_{1}+\left(W_{1}-W_{2}\right) / R_{\text {gap }}=I_{1} \\
W_{k} / Z_{k}+\left(W_{k}-W_{k-1}\right) / R_{\text {gap }}=I_{k}+\left(W_{k+1}-W_{k}\right) / R_{\text {gap }} \quad k=2,3, \ldots, n-1 \\
W_{n} / Z_{n}+\left(W_{n}-W_{n-1}\right) / R_{\text {gap }}=I_{n}
\end{array}\right.
$$

For the sake of simplicity, junctional coupling was assumed to be homogenous across the network, while individual impedances were different. These choices do not affect the generality of our conclusions.

Equation (7) generalizes the analysis proposed in Amitai et al. (2002) to the case of persistent sinusoidal regimes (AC), and follow from imposing the conservation of charge at each node. By definition, impedances $\left\{Z_{k}\right\}$ take the form of R-C parallels,

$Z_{k}(\omega)=\left[R_{m} /\left(j \cdot \omega \cdot R_{m} \cdot C_{m}+1\right)\right]_{k}$.

If only one cell (i.e. the $\mathrm{m}$-th cell) is being stimulated at a time by current injection (i.e. $I_{k}=0, k \neq m$ ), simple transfer relationships between neighboring $W_{k}$ can be derived from Eq. (7):

$$
\begin{aligned}
W_{k+1} & =F_{k+1} W_{k} \\
\mathrm{k} & =(\mathrm{n}-1),(\mathrm{n}-2), \ldots,(\mathrm{m}+1), \mathrm{m} \\
W_{h-1} & =B_{h-1} W_{h} \quad \mathrm{~h}=2,3, \ldots,(\mathrm{m}-1), \mathrm{m}
\end{aligned}
$$

These relationships, referred to as "forward" $F_{k}$ and "backward" $B_{h}$ depending on the location of the recorded cells with respect to the site of current injection, are complex functions of $\omega$ and are iteratively computed from Eq. (7), as

$$
\begin{aligned}
& \left\{\begin{array}{l}
F_{n}=Z_{n} /\left(Z_{n}+R_{\text {gap }}\right) \\
F_{k}=Z_{k} /\left[R_{\text {gap }}+Z_{k} \cdot\left(2-F_{k+1}\right)\right] \quad k=(n-1),(n-2), \ldots,(m+2),(m+1)
\end{array}\right. \\
& \left\{\begin{array}{l}
B_{1}=Z_{1} /\left(Z_{1}+R_{\text {gap }}\right) \\
B_{h}=Z_{h} /\left[R_{\text {gap }}+Z_{h} \cdot\left(2-B_{h-1}\right)\right] \quad h=2,3, \ldots,(m-2),(m-1)
\end{array}\right.
\end{aligned}
$$

$F_{k}$ and $B_{h}$ summarize the AC signal transfer properties between neighboring cells in a compact form. Their "cascade" makes possible to analyze the filtering properties of the entire chain or of a part of it in the frequencydomain. From Eq. (8) it follows immediately that for large $\omega, \mathrm{F}_{n}$ and $\mathrm{B}_{1}$ can be approximated as $\sim(\mathrm{j} \omega)^{-1}$. By means of symbolic and numerical evaluation of Eqs. (11) and (12) (not shown), the very same result holds for $F_{k}$ and $B_{h}$, so that

$$
\left\{B_{1}, B_{2}, \ldots, B_{m-1}, F_{m+1}, F_{m+2}, \ldots, F_{n}\right\} \sim(j \cdot \omega+2 \pi \cdot p)^{-1} .
$$

This indicates that each transfer function is dominated by the frequency response of a system with a single timeconstant $p^{-1}$ (i.e. by a single transmission "pole" $p$ ). The location of $p$ on the frequency axis results from a combination of the values of $\left\{\mathrm{R}_{\mathrm{m}}, \mathrm{C}_{\mathrm{m}}\right\}_{\mathrm{k}}$ and $\mathrm{R}_{\text {gap }}$.

In general, defining as $W_{i}=Q_{i j} W_{j}$ the transfer relationships between non-adjacent cells of the network (e.g. $|\mathrm{i}-\mathrm{j}|$ cells far apart), it can be shown that

$$
\begin{aligned}
\mathrm{i}<\mathrm{j} \leq \mathrm{m} \quad \mathrm{Q}_{\mathrm{ij}}=\mathrm{B}_{\mathrm{i}} \cdot \mathrm{B}_{\mathrm{i}+1} B_{\mathrm{i}+2} \cdot \ldots \cdot \mathrm{B}_{\mathrm{j}-2} \\
\cdot \mathrm{B}_{\mathrm{j}-1} \underset{\omega \rightarrow+\infty}{\longrightarrow}(\mathrm{j} \cdot \omega)^{-(\mathrm{j}-\mathrm{i})}
\end{aligned}
$$




$$
\begin{aligned}
\mathrm{i}>\mathrm{j} \geq \mathrm{m} \quad \mathrm{Q}_{\mathrm{ij}}= & \mathrm{F}_{\mathrm{i}} \cdot \mathrm{F}_{\mathrm{i}-1} \mathrm{~F}_{\mathrm{i}-2} \cdot \ldots \cdot \mathrm{F}_{\mathrm{j}+2} \\
& \cdot \mathrm{F}_{\mathrm{j}+1} \underset{\omega \rightarrow+\infty}{\longrightarrow}(\mathrm{j} \cdot \omega)^{-(\mathrm{i}-\mathrm{j})}
\end{aligned}
$$

since the frequency response of a cascade of linear systems is equivalent to the product of individual frequency responses (Brogan 1991). Equations (14) and (15) state that connection proximity between two cells (e.g. $\left.d_{j}(i)=|i-j|\right)$, can be inferred from the high-frequency slope of the magnitude of a complex transfer function $Q_{i j}$ or from its asymptotic phase shift.

Although $F_{k}$ and $B_{h}$ have an experimentally identifiable correlate as transfer-impedances of neighboring cells, distinguishing among them without a prior knowledge of the 1-d network topology (i.e. $m, k$ and $h$ ) is not possible. Indeed, if $\mathrm{i}<\mathrm{m}<\mathrm{j}$ or $\mathrm{j}<\mathrm{m}<\mathrm{i}$, one can anticipate ambiguities in inferring the connection proximity, because of zero-pole cancellations. In fact,

$\mathrm{i}<\mathrm{m}<\mathrm{j} \quad Q_{i j}=\frac{B_{i} \cdot B_{i+1} B_{i+2} \cdot \ldots \cdot B_{j-2} \cdot B_{m-1}}{F_{j} \cdot F_{j-1} \cdot F_{j-2} \cdot \ldots \cdot F_{m+2} \cdot F_{m+1}} \underset{\omega \rightarrow+\infty}{\longrightarrow}(j \cdot \omega)^{-(i+j-2 \cdot m)}$.

Symbolic electrical network analysis as well as extensive computer simulations of 1-dimensional chains of singlecompartmental conductance-based neurons confirmed the existence of this ambiguity. For instance, in a small network composed by 4 neurons, where only the second one was stimulated (m=2), $Q_{14}$ could be approximated with good accuracy by a single term $\sim(j \omega)^{-1}$ and not by $\sim(j \omega)^{-3}$, since $(i+j-2 m)=1$, although $(j-i)=3$.

Operatively, this situation can be avoided by performing current injection in one cell (i.e. the m-th) at a time, and recording the membrane potentials of any other cell (i.e. the $\mathrm{k}$ - $t h$, with $\mathrm{k} \neq m$ ). The transfer impedance will then be computed as the ratio between Fourier transforms of the voltage traces recorded in the two cells (Brogan 1991): $\mathrm{W}_{\mathrm{k}} / \mathrm{W}_{\mathrm{m}}$.

\section{2-Dimensional networks of electrically coupled neurons and resistive grids}

Does the theoretical result demonstrated in the previous section hold for more complex network topologies? Here we considered an infinite and homogeneous 2-dimensional network of passive single-compartmental neurons, each of them electrically coupled to its four nearest-neighbors by gap-junction of identical conductance [Fig. 5(a)]. In this section, we test and verify the hypothesis that the highfrequency properties of the transfer impedance between any two cells of this network could take the form of a powerlaw exponent, coincident with the shortest-path city-block distance, also known as Manhattan-distance. We studied analytically these infinite networks by a different mathematical strategy, taking advantage of the lack of boundaries. Results have been successfully verified for small finite 2D topologies by means symbolic electrical networks analysis, suggesting that boundary conditions have no effect and that our method indeed depends only on the shortest-path.

Let's consider a 2-dimensional infinite resistive grid (Atkinson and van Steenwijk 1999; Venezian 1994), where each element has identical conductance $\mathrm{G}=\mathrm{R}_{\text {gap }}^{-1}$, modeling the electrical coupling between a cell and its four nearest-neighbors. Each node of this grid corresponds to the intracellular space of a single-compartmental neuron so that
Fig. 5 Infinite networks of identical (passive) single-compartmental neurons. This case was studied under the hypothesis of homogeneous 2-dimensional nearest-neighbor electrical coupling (a), as an AC variant of resistive lattices (b). The connection proximity $d$ between an injected cell and another one can be estimated by our AC method and it coincides with the cityblock or Manhattan-distance
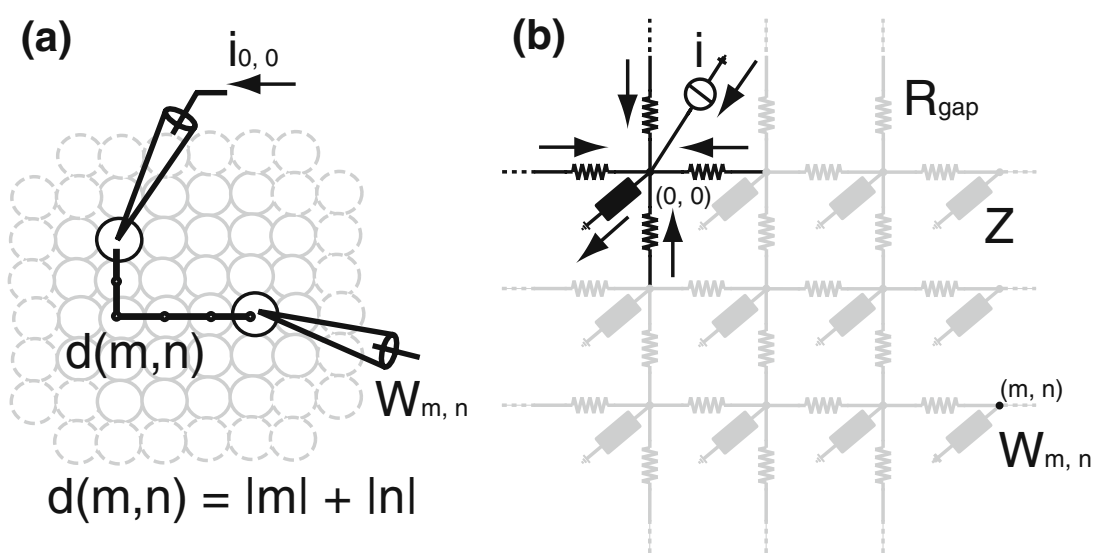
the electrical potential $W_{m, n}$ at each node represents the transmembrane potential of the neuron located at coordinates $(m, n)$ (Fig. 5). Each node is also connected to ground through impedances $Z$, which account for the capacitive and resistive properties of the cell membrane as in Fig. 4 [see also Eq. (8)]. These impedances have been made identical for the sake of analytical simplicity. At each node, we further consider an external current injection $I_{m, n}$. Figure 5 generalizes to the 2-dimensional case the model represented in Fig. 4, for an infinitely extended uniform network.

Since the AC behavior of the network is our main focus, we indicate by $W$ and $I$ the Fourier transforms of voltages and injected currents at each node [see also Eq. (6)], as we did for the 1-dimensional case. We can now write the charge conservation at a generic node $(m, n)$ as

$$
\begin{aligned}
I_{m, n} & +\left(W_{m+1, n}+W_{m-1, n}+W_{m, n+1}+W_{m, n-1}-4 \cdot W_{m, n}\right) \cdot G \\
& =W_{m, n} / Z
\end{aligned}
$$

Exploiting the symmetries of the system and the lack of boundaries, we approach the solution of Eq. (17) by (spatial) spectral methods. The same approach can be used for the 1-dimensional case, confirming the theoretical results presented in the previous section in the case of an infinite 1D network (not shown). We recall the 2-dimensional discrete-space Fourier transform pairs as

$$
\begin{aligned}
I_{m, n} & =\frac{1}{4 \cdot \pi^{2}} \int_{-\pi}^{\pi} \int_{-\pi}^{\pi} d u \cdot d v \cdot I(u, v) \cdot e^{j \cdot(m \cdot u+n \cdot v)} \\
I(u, v) & =\sum_{m=-\infty}^{+\infty} \sum_{m=-\infty}^{+\infty} I_{m, n} \cdot e^{-j \cdot(m \cdot u+n \cdot v)} \\
W_{m, n} & =\frac{1}{4 \cdot \pi^{2}} \int_{-\pi}^{\pi} \int_{-\pi}^{\pi} d u \cdot d v \cdot W(u, v) \cdot e^{j \cdot(m \cdot u+n \cdot v)} \\
W(u, v) & =\sum_{m=-\infty}^{+\infty} \sum_{m=-\infty}^{+\infty} W_{m, n} \cdot e^{-j \cdot(m \cdot u+n \cdot v)} .
\end{aligned}
$$

If an external AC current of (complex) amplitude $i$ is injected only at a single node at a time, whose coordinates can be always set to $(0,0)$ (i.e. $\left.\mathrm{I}_{m, n}=\mathrm{i} \delta_{m, n}\right)$ by a change of coordinates [see Fig. 5(b)], the difference Eq. (17) can be rewritten equivalently as an algebraic relation, in the complex variables $\mathrm{u}, \mathrm{v}$, and $\omega$ :

$i+\left(e^{j \cdot u}+e^{-j \cdot u}+e^{j \cdot v}+e^{-j \cdot v}-4\right) \cdot G \cdot W(u, v)=W(u, v) / Z$.
Equation (20) can be further rearranged, solving it for W(u, $\mathrm{v})$. Then the transfer function between the AC current $i$ injected in the node with coordinates $(0,0)$ and the $\mathrm{AC}$ voltage recorded at the generic node $(\mathrm{m}, n)$ requires an antitransformation as in Eq. (19):

$$
\begin{aligned}
T_{m, n}= & \frac{W_{m, n}}{i}=\frac{1}{4 \cdot \pi^{2}} \int_{-\pi}^{\pi} \int_{-\pi}^{\pi} d u \cdot d v \\
& \cdot\left[\frac{1}{2 \cdot G \cdot(\cos u+\cos v-2)-Z^{-1}}\right] \cdot e^{j \cdot(m \cdot u+n \cdot v)}
\end{aligned}
$$

We note that the transfer function that we are considering here is between the membrane voltage $\mathrm{W}_{m, n}$ of a generic node and the current $i$ injected at $(0,0)$, instead of the corresponding membrane voltage $\mathrm{W}_{0,0}$. This is only related to considerations on analytical simplicity and convenience and makes the inference on the connection proximity from high-frequency power-law exponent of Eq. (21), off of exactly one unit. In fact, the transfer impedance $\mathrm{W}_{m, n} / \mathrm{W}_{0,0}$ can be decomposed by linearity as the product between $\mathrm{T}_{m, n}=\mathrm{W}_{m, n} / \mathrm{i}$ and $\mathrm{Z}^{-1}=\mathrm{i} / \mathrm{W}_{0,0}$, where $\mathrm{Z}$ is the membrane impedance of the node $(0,0)$. Because by definition $\mathrm{Z} \sim(\mathrm{j} \omega)^{-1}$ [Eq. (8)], in order to estimate the power-law exponent $d$ of the impedance between $\mathrm{W}_{m, n}$ and $\mathrm{W}_{0,0}$, the absolute exponent of $\mathrm{T}_{m, n} \sim(\mathrm{j} \omega)^{-(\mathrm{d}+1)}$ must be decreased of one unit. We also note that the mathematical expressions for $\mathrm{T}_{m, n}$ and $\mathrm{T}_{n, m}$ are equivalent [see Eq. (21)], as expected from symmetry considerations.

As the integral of Eq. (21) cannot be evaluated in closed form, we approached it numerically, by means of the adaptive Simpson quadrature numerical method available in MATLAB, after making explicit the dependence of $\mathrm{Z}$ on $j \omega$ (i.e. $Z^{-1}=\mathrm{R}_{m}^{-1}+j \omega \mathrm{C}_{m}$ ). For each value of $\omega$, $\mathrm{T}_{m, n}(\omega)$ is a complex number whose magnitude and phase provide the gain and the phase shift between the (current injected at) node $(0,0)$ and the (voltage recorded at) node $(m, n)$. As plotted in Fig. 6 for a few combinations of pairs $(m, n)$, at high frequency $\mathrm{T}_{m, n} \sim(\mathrm{j} \omega)^{-\alpha}$ with $\alpha$ integer and coinciding with the Manhattan-distance between $(m, n)$ and $(0,0)$ plus "1", for the reasons clarified above. We finally note that the tolerance of the numerical double integration had to be lowered when large values of $(m, n)$ were considered, consistent with the correspondingly stronger transfer attenuations obtained.

Concluding, these analytical/numerical results demonstrate that $\mathrm{T}_{m, n} \sim(\mathrm{j} \omega)^{-(|m-0|+|n-0|+1)}$, and that it can be employed to infer the (shortest) distance between cells $(m, n)$ and $(0,0)$, in 2-dimensional networks with nearestneighbor connectivity. 

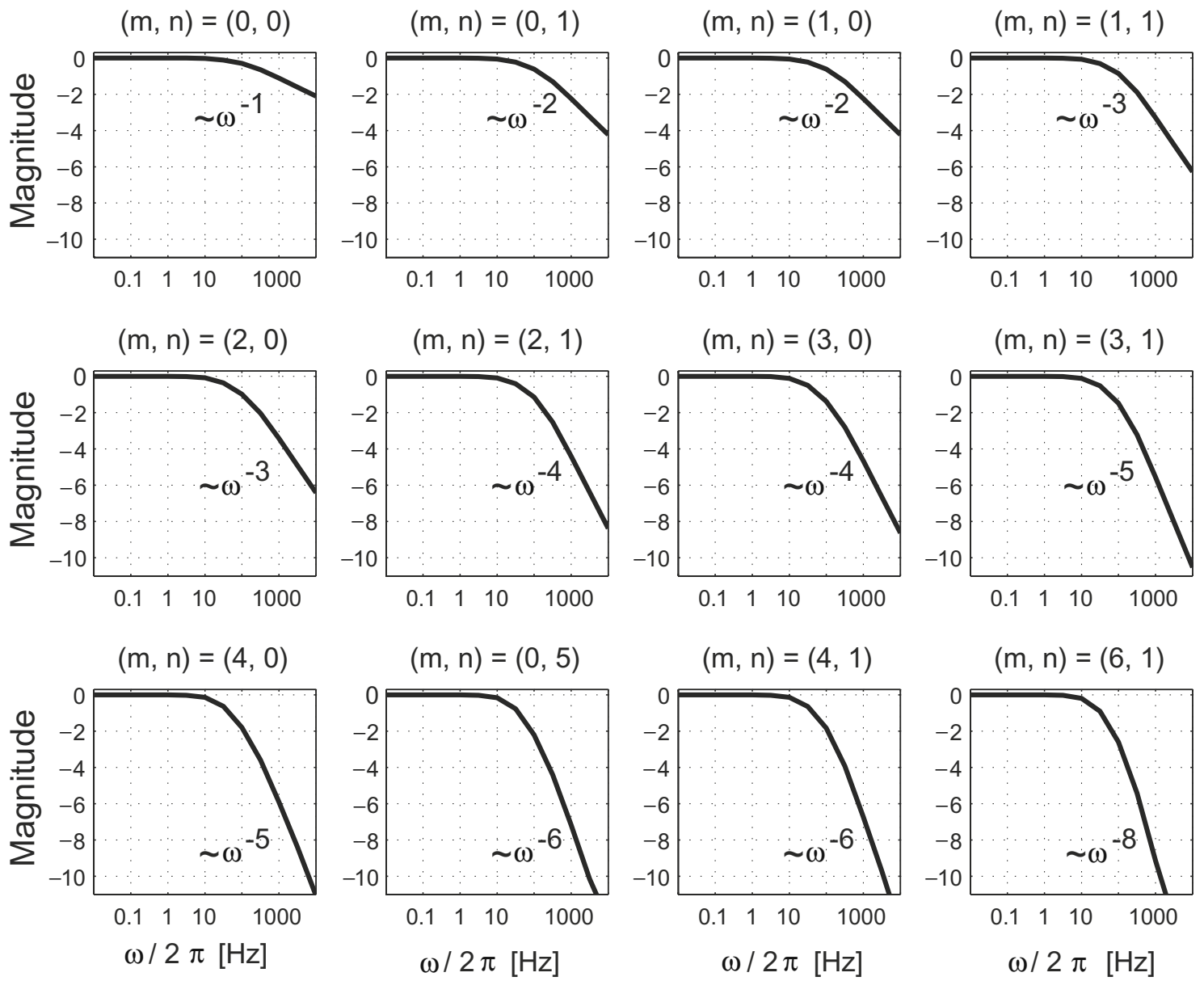

Fig. 6 Shortest (city-block) distance in infinite 2-dimensional networks. The transfer impedance $\mathrm{T}_{m, n}$ between the AC injected current $i$ and the Fourier transform of the voltage $\mathrm{W}_{m, n}$ at a generic node $(m, n)$ could be computed analytically for homogenous impedances $Z=R C$ and junctional coupling G [Fig. 5(b), Eq. (21)]. By numerical integration reported in the subplots for different pairs $(m, n)$, it is apparent that $\mathrm{T}_{m, n}$ takes the form of a power law $(j \omega)^{-\alpha}$. The exponent $\alpha$ is integer and it is proportional to the shortest city-block

\section{Multi-compartmental models}

A full characterization of the impact of morphological anatomical complexity to the frequency-dependent signal transduction of gap-junctions (Prinz and Fromherz 2003) was beyond our goals. However, as a first step, we considered the Rall's multicompartmental model (Dayan and Abbott 2001) to explore limitations of our assumptions. A compact soma region was connected to an equivalent cylindrical cable, replacing a complex dendritic tree. Axons were not modeled and axo-somatic, axo-dendritic and axoaxonic gap-junctions were ignored as they are thought to play a minor role for subthreshold voltages. For instance, cortical GABAergic interneurons are usually coupled somatically or dendritically, as revealed by ultrastructtural studies (Tamás et al. 2000; Szabadics et al. 2001).

Gap-junctions between neighboring model cells were then located somatically or at different locations on the cylinder.

As expected, when cable structures of significant length are introduced between symmetrical dendro-dendritically coupled model neurons, which are recorded and currentinjected exclusively at the soma, our method fails. Standard $\mathrm{AC}$ analysis of the cable equation, as reported in the following section [see Eq. (24)], confirmed that a linear dynamical system with distributed parameters such as a passive cable, cannot be described in terms of an integer number of zeros and poles (i.e. an integer power-law exponent) and as a finite phase-shift (Fig. 7). Indeed, the frequency-independent delays and the fractional lengthdependent power-law behavior violate our assumptions on the correspondence "connection proximity=filter order", as soon as the length of the cable or the somatodendritic 
Fig. 7 Quantification of the method limitations to electrotonically compact cells. Cable $\mathrm{AC}$ impedance has been computed at three distinct spatial locations (i.e. $25 \%, 50 \%$, and $100 \%$ of the cable length) and for different cable lengths, indicated as multiples of the cable space constant $\lambda$. The gray boxes outline the range of frequencies employed in our previous ZAP analysis. Red traces are subplots of integer powerlaw functions $(\omega)^{-\alpha}(\alpha=1,2,3)$, which appear as lines of negative slope in the double logarithmic scale, and which have been plotted for the sake of comparison with the "effective" local slopes of the cable impedance magnitude
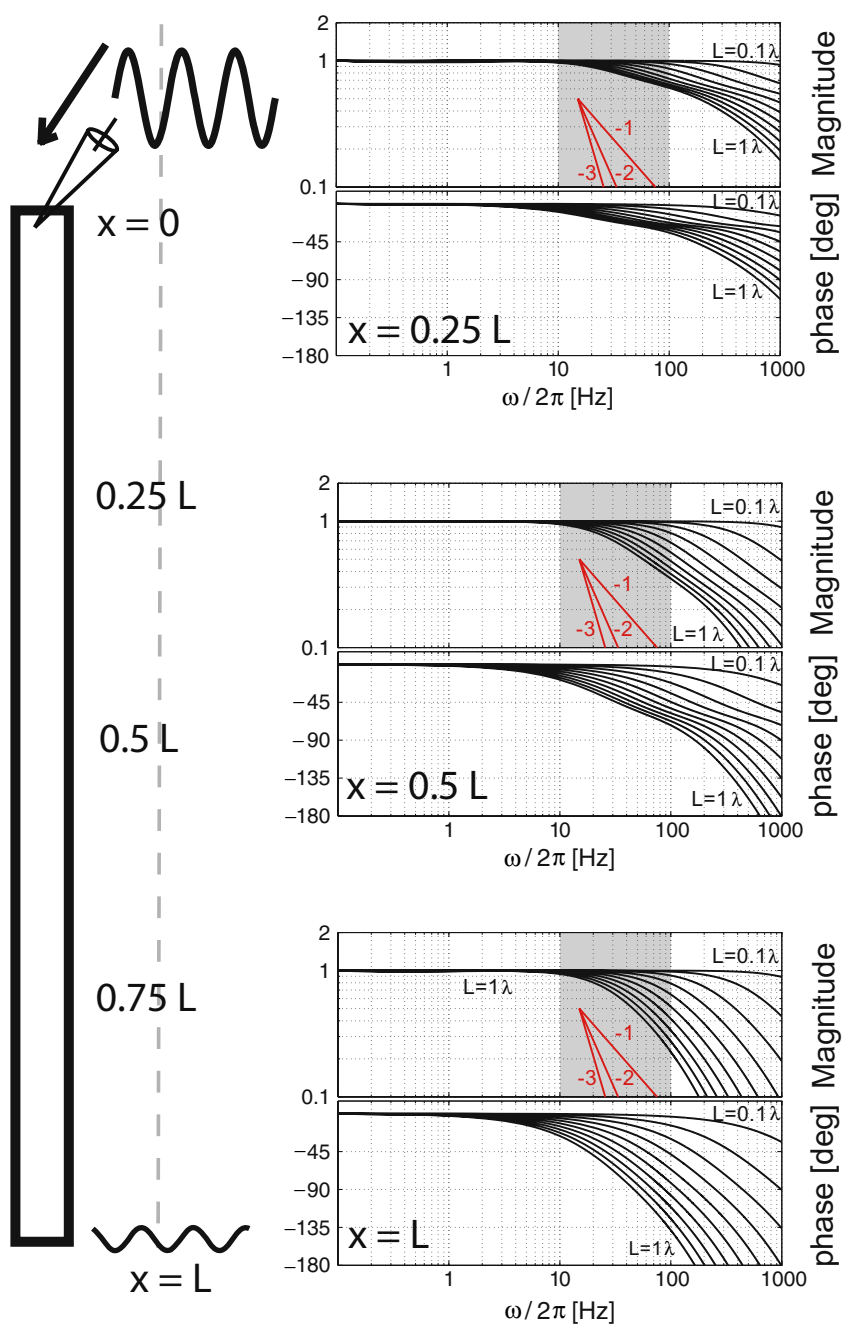

distance of the gap-junctions become comparable to the cylinder space constant. We quantified such limitations by comparing the effective slope of the (cascade) transfer properties induced by the cable: as soon as this slope becomes comparable or steeper than $(\mathrm{j} \omega)^{-1}$, our method will erroneously conclude that an intermediate cell is present between directly coupled cells. On the other hand, for short cables or proximal gap-junctions, our conclusions are affected only at very high frequencies (e.g. larger than $500 \mathrm{~Hz}$ ), making possible to interpret correct results from our method and to obtain correct answers through the fit procedure, at least in the intermediate frequency range considered in the numerical simulations.

\section{$7 \mathrm{AC}$ analysis of the cable equation}

When the hypothesis of space-clamp is inappropriate, passive cable theory (Weiss 1996) can be considered to identify the limits of our approach. Neglecting active membrane currents, we recall that the spatio-temporal distribution of the membrane voltage $V(x, t)$ on a uniform cable of length $L$, can be described by the following equation (Rall 1969):

$\lambda^{2} \cdot \frac{\partial^{2} V(x, t)}{\partial x^{2}}=\tau_{m} \cdot \frac{\partial V(x, t)}{\partial t}+V(x, t)$,

where $\tau_{\mathrm{m}}$ and $\lambda$ are the time and space constants of the cable, respectively (Dayan and Abbott 2001). These constants are related to geometrical (i.e. the radius a) as well as electrical properties of the cable (i.e. the specific membrane capacitance $\mathrm{c}_{\mathrm{m}}$, the specific transmembrane resistance $r_{m}$ and the cytoplasmic axial resistivity $r_{a}$ ) as follows,

$\lambda^{2}=0.5 \cdot a \cdot r_{m} / r_{a}$ and $\tau=r_{m} \cdot c_{m}$.

Under stationary sinusoidal regime, the partial differential Eq. (22) become an ordinary one, upon Fouriertransforming both sides [see Eq. (6)]

$\frac{d^{2} W(x, j \omega)}{d x^{2}}=\frac{1}{\lambda_{\omega}^{2}} \cdot W(x, j \omega)$, 
where $W(\mathrm{x}, \omega)$ is the Fourier transform of the membrane voltage $V(\mathrm{x}, \mathrm{t})$ [see Eq. (6)]. It is apparent that the solutions of Eq. (23) in the frequency domain are formally analogous to the steady-state solutions of Eq. (22) in the time-domain, where $\lambda$ is replaced by $\lambda_{\omega}=\lambda /\left(1+\tau_{\mathrm{m}} \mathrm{j} \omega\right)^{0.5}$ being a complex number depending on the angular frequency $\omega$.

For a cable of finite length $L$, characterized by a "sealed end" at $x=L$ (Weiss 1996) and by an external current injection at $x=0$, the AC transfer properties between the site of injection (i.e. $x=0$ ) and a generic point at the coordinate $x$ can be expressed as [similarly to Eqs. (14), (15), and (21)]

$$
\frac{W(x, j \omega)}{W(0, j \omega)}=\frac{\cosh \left[(L-x) / \lambda_{\omega}\right]}{\cosh \left(L / \lambda_{\omega}\right)} \quad 0<x<L
$$

For convenience, $\lambda_{\omega}$ is expressed as $\lambda_{\omega}=M e^{j \phi}$, or equivalently as $\lambda_{\omega}=a+j b$, with

$$
\begin{aligned}
M & =\lambda \cdot\left(1+\tau_{m}^{2} \cdot \omega^{2}\right)^{-0.25} \quad \varphi=-0.5 \cdot \operatorname{arctg}\left(\tau_{m} \cdot \omega\right) \\
a & =M \cdot \cos (\varphi) \quad b=M \cdot \sin (\varphi) .
\end{aligned}
$$

Figure 7 reports the study of Eq. (24), where magnitude and phase of the cable transfer impedance have been plotted over a wide range of frequencies, at three distinct spatial locations (i.e. $25 \%, 50 \%$, and $100 \%$ of the full length $L$ ) along cables of various lengths. Each subplot summarizes the impact of cable properties, isolated from the soma, to be considered for distal gap-junctions. The overall transfer impedance between electrically coupled cells is then multiplicatively corrupted by the impedance of the cable. Within the intermediate frequency range $[10 ; 100] \mathrm{Hz}$, the

(a)
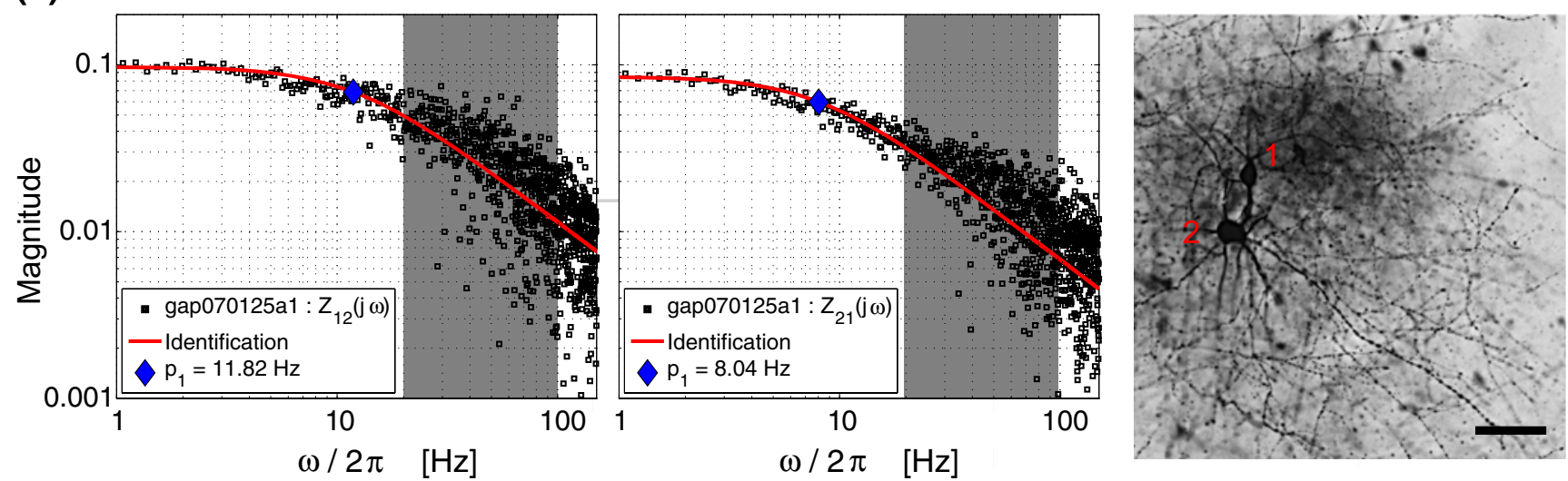

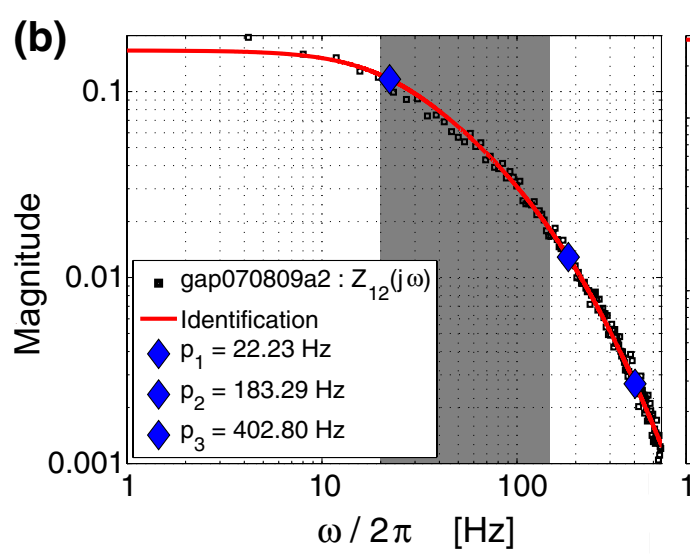

Fig. 8 Preliminary test of the method on electrically coupled layer 6 cortical interneurons. Two cells patched simultaneously revealed to be electrically connected (a) and were stained by biocytin (right-scale: $60 \mu \mathrm{m})$. Although the staining was very strong, no dye-coupling to intermediate cells was observed. Best-fit of Eq. (5) indicates that the transfer-impedances $\mathrm{Z}_{12}$ (left) and $\mathrm{Z}_{21}$ (center) had a unitary powerlaw exponent, suggesting a direct connection. In another experiment (b), as the effect of spontaneous chemical synaptic transmission was pharmacologically blocked, the signal-to-noise ratio was extremely

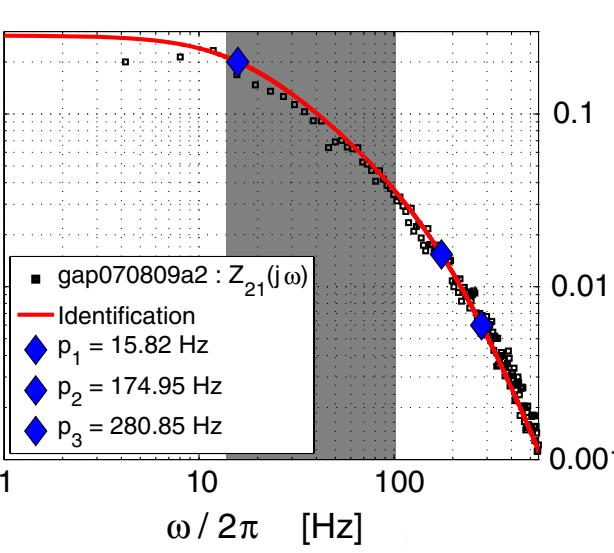

favorable and the transfer-impedances between two interneuons could be tested up to $500 \mathrm{~Hz}$. Best-fit of Eq. (5) indicates a unitary powerlaw in the intermediate frequency range and a third-order power-law for very high frequency. Although no morphological staining is available, from comparison with the plots of Fig. 7 and given the relatively large DC coupling strength (i.e. $\sim 0.16-0.28$ ), cable filter properties are more likely to account for the data than intermediate cells (right) 
maximal slope can be up to -3 for a cable of length $L=3 \lambda$, while recording at the maximal distance $\mathrm{x}=\mathrm{L}$. Under the same conditions, for $\mathrm{x}=0.25 \mathrm{~L}$, the maximal slope is $\sim-1$, and for $\mathrm{x}=0.5 \mathrm{~L}$ it is $\sim-2$.

These considerations suggest that if a gap-junction is located distally in a structure that is not electrotonically compact, it is not possible to infer reliably the connection proximity from the integer slope of the cell-to-cell transfer impedance: not only the slope might become fractional instead of integer but its value can be affected by an "offset", introduced by neuritic cable properties and not by cell-to-cell proximity.

\section{Experimental results for neocortical interneurons}

We performed preliminary experiments in rat neocortical acute slices, recording from layer 6 interneurons (see the Methods) while injecting ZAP waveforms as current-clamp stimuli [Eqs. (1-3)]. Because of the usually small coupling coefficients as reviewed by Hestrin and Galarreta (2005), stable recording conditions and averaging over repeated identical stimulation trials $(\sim 50-100)$ were necessary conditions for a good signal-to-noise ratio as in the investigation of very weak postsynaptic potentials. Spontaneous (chemical) synaptic activity was pharmacologically blocked to improve baseline voltage stability. Figure 8 reports the transfer impedances estimated and analyzed by the very same tools described in the text and employed in Fig. 3 for simulated data. For the cell pair of top panels Fig. 8(a), the high-frequency power-law exponent could be approximated with good accuracy by 1 , through best-fit of Eq. (5). Therefore, our method suggests that a direct electrical connection existed between those interneurons. The transmitted-light microscopy photograph of biocytin staining neuronal morphologies [Fig. 8(a) -right panel] independently supports such a conclusion, revealing a close spatial arrangement of the cells and no dye-coupled intermediate neuron (see Dupont et al. 2006).

In another experiment, unusually optimal recording conditions prompted us to test much higher ZAP frequencies, up to $500 \mathrm{~Hz}$. Best-fit of Eq. (5) revealed an excellent agreement with the data, requiring 3 poles whose location was unexpectedly spread across the frequency axis, as opposed to the simulated data of Fig. 3. However in this particular case, because of the large coupling coefficient (i.e. above 0.1 ) and the relatively close arrangement of the patch-pipettes (not shown), it is perhaps unlikely that three intermediate cells were involved. Instead, a relatively short cable filtering (see Fig. $7-$ e.g. $x=0.25, L=0.5 \lambda$, or e.g. $x=L, L=0.3 \lambda$ ) could account for the data, indicating that the junctional coupling was not proximal in at least one of the two cells.

\section{Discussion}

In this work we introduced a simple technique, based on the framework of linear circuit theory. We exploited the properties of electrical synapses in attenuating signals from one cell to another in a frequency-dependent way, similar to what is expected from filter cascades. Frequency-dependent properties of gap-junctions have been already observed experimentally by Hestrin and Galarreta (2005). Here, we exploited the quantitative properties of this (low-pass) behavior for inferring microcircuit-level connectivity. The method relies on the strong assumption of electrotonic compactness or of junctional coupling proximal to the recording locations. Under a similar perspective, the use of appropriate channel blockers in the intracellular pipette solution or bath-applied might approximate our assumptions in real experiments. In particular, sodium and potassium-currents blockade by lidocaine derivates (e.g. QX314) or by Tetrodotoxin (TTX), as well as the blockade of H-currents (e.g. ZD7288) could be used in case the conditions of a network of passive elements are violated by strong subthreshold resonances. In addition, a variety of pharmacological tools can be differentially employed to manipulate the gap-junctions (Salameh and Dhein 2005). When the mapping of electrical connectivity is of interest, these tools can be exploited to facilitate the recording conditions by means of unphysiologically increasing the junctional conductance and improving the signal-to-noise ratio as in our simulations.

Our theoretical results are general [Eqs. (8), (14), (15), and (21)] and they do not depend on the values chosen for the junctional coupling. Similarly, semi-analytical circuit analysis confirmed the validity of our method for both heterogeneous and homogeneous networks, as well as for symmetrical and asymmetrical simple topologies (not shown). However, in the simulations of Figs. 1, 2 and 3 and in the numerical integration presented in Fig. 6 we chose unphysiological large coupling coefficients. As mentioned in Section 3, the location but not the number of poles $\{p\}_{i}$ of the transfer impedances results from a combination of the intrinsic membrane properties (i.e. $\mathrm{R}_{\mathrm{m}}, \mathrm{C}_{\mathrm{m}}$ ) and of the junctional coupling $R_{\text {gap }}$. In a 2-cells network, it is easy to show that the smaller $R_{\text {gap }}$ the higher signal-to-noise ratio, as intuitively expected, but also the higher the location of $\{p\}_{i}$ on the frequency-axis. On the contrary, for a very large junctional resistance the coupling coefficient goes to zero and the location of the $\{p\}_{i}$ approaches the inverse of the membrane time constants. These observations imply that 
our choice for the simulation parameters did not compromise the generality of our conclusions.

Although we have preliminary applied our method in real electrophysiological experiments (Fig. 8), its general applicability and confidence remain to be quantified. In fact, as already mentioned, (biocytin) staining alone [Fig. 8(a)] cannot be fully conclusive as an independent method of cross-validation for electrical connectivity. Nevertheless, even though we overdrove the ABC staining procedure (Hsu et al. 1981) to maximize the chance for the dye to diffuse through gap junctions, no additional cell appeared stained in the experiment reported by Fig. 8(a). Indeed, Dupont et al. (2006) showed that patching neurons with a biocytin-filled patch-clamp electrode might result in filling up to 15 dye-coupled neurons.

A drawback of the method is certainly the sensitivity to measurement and biological noise. In fact, for very weak electrical coupling and indirect connectivity, the frequencydependent low-pass attenuation considerably makes the estimation of the transfer-impedance profile more difficult as the evoked postsynaptic oscillations are buried in the noise. Then, extensive response averaging over repeated identical trials $(\sim 50-100)$ and slower $(\sim 20-30$ s), longerlasting, ZAP frequency sweeps become important requirements. In fact, assuming that additive noise corrupts the evoked voltages, its fluctuations uncorrelated to the ZAP waveform will be averaged away. In addition, longer voltage responses evoked by slower ZAP frequency-sweeps will increase the resolution of the transfer impedance in the frequency-domain, as estimated by discrete Fourier techniques (Oppenheim et al. 1998). However, by similar mathematical tools as those we employed it might be possible to refine and extend our approach to simultaneous multiple current injections with independent waveforms, possibly improving the signal-to-noise ratio as a result of the stronger overall stimulation.

In a real experimental setting, the finite quantization precision of Analog/Digital data acquisition boards (e.g. 14bits) together with a typical dynamical range after amplification (e.g. $\pm 100 \mathrm{mV}$ ) set a lower limit to the detection of postsynaptic responses (i.e. $200 \mathrm{mV} / 2^{14} \sim 10 \mu \mathrm{V}$ ) and thus an upper bound on the connection proximity (i.e. 2-3 intermediate cells) that can be observed. Such an estimate assumes a direct coupling coefficient not larger than $10 \%$ (Galarreta and Hestrin 2001) and subthreshold presynaptic ZAP amplitude of $5-10 \mathrm{mV}$. It is interesting to note that the possibility of observing a maximum of $0-2$ intermediate cells might be still adequate for discriminating between certain statistical models of connectivity (see Fig. 9), as a first step in tackling (e.g.) the patchy-vs-syncytium problem for interneuronal electrical synaptic organizations.

We note that the method presented here is approximately equivalent in the time domain to separating the distinct

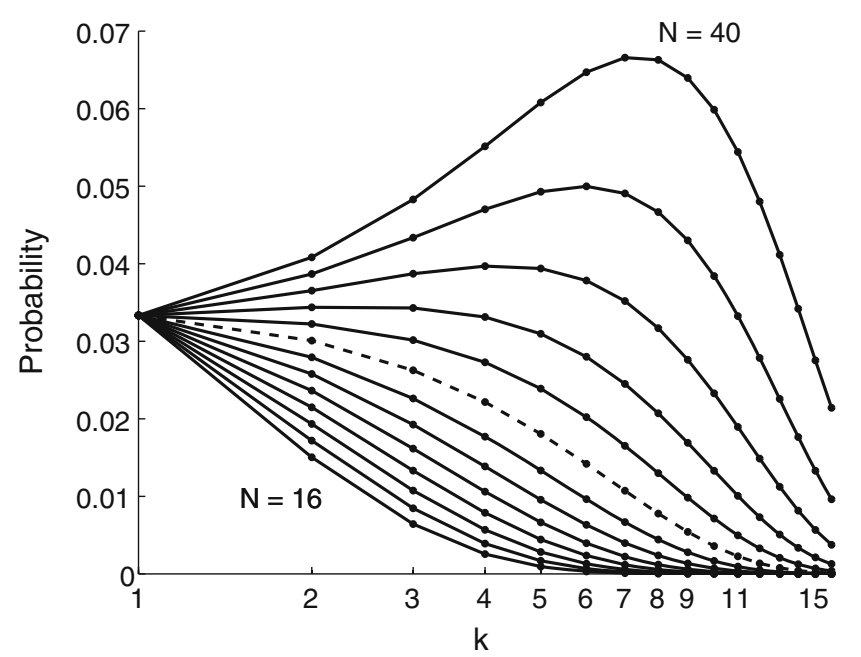

Fig. 9 Theoretical distribution of (shortest) connection proximities for a randomly fractured network. Equation (28) has been plotted setting the probability $(1-q)=30^{-1}$, for different values of connection proximity $k$ and different network sizes $N$. The dotted curve corresponds to $N=30$ and represents on a first approximation the threshold for a phase-transition, as expected in Erdős-Rényi random graph models

time-constant in the trans-neuronal voltage responses to a step current stimulus. However, the characterization of the transfer impedance, here proposed to map electrical connectivity, might constitute a direct way to experimentally explore network-level properties. For instance, subthreshold transfer properties and resonances arising from inter-neuronal active mechanisms (Saraga et al. 2006) might be investigated in the frequency-domain. In fact, distinct voltage-gated channels from distinct neurons might interact through electrical coupling, in close analogy to those subthreshold resonances that arise from single-cell membrane properties.

The method discussed here generalizes to time-varying regimes the tools employed in Amitai et al. (2002) that quantified the impact of direct and indirect electrical coupling on the passive properties of single neurons at the steady-state. Similarly to that study, compact electrotonic morphologies or junctions proximal to the recording sites are necessary conditions to allow general mathematical treatment. For these reasons, the method might be applied with success especially to decipher the electrical- and the chemical-signaling connectivity of small cortical interneurons or astrocytes (Wallraff et al. 2006; Bennett and Zukin 2004). From the results of Fig. 8(b), showing a segregation of the best-fit poles location, it is perhaps routinely possible to indirectly infer the presence of substantial cable filtering. Alternatively, kinetic analysis of miniature synaptic potentials might further provide partial indirect indications on the electrotonical compactness of neurons and thus on the confidence of our method for real applications. As recently 
examined for cortical as well as hippocampal interneurons (Dumitriu et al. 2007; Cossart et al. 2006) the analysis of the rise time, decay time and amplitude of spontaneous synaptic events recorded under TTX, revealed the existence of interneuronal classes such as the parvalbumin-positive GABAergic neurons (basket cells), which might then be ideal targets to characterize electrical connectivity through our protocol.

Nevertheless, distinct electrophysiological techniques, such as somatodendritic recordings or voltage-sensitive dye imaging (reviewed in Davie et al. 2007 and Stuart and Palmer 2006, respectively) could overcome the limitations described so far because distal voltage recordings might complement somatic ones (Stuart and Palmer 2006; Djurisic et al. 2004). In cases where cable properties cannot be neglected, the experimental evidence of a fractional power-law exponent constitutes a sufficient condition for inferring a distal dendritic location of gap junctions (e.g. in mitral cells - see Migliore et al. 2005). Along the same motivations of Prinz and Fromherz (2003), these observations could be turned into quantitative geometrical configurations by fitting the $\mathrm{AC}$ solutions of the cable equation [Eq. (24)].

Finally, interesting analogies can be drawn between studies on AC and DC "hopping conduction" in physical media and the generalization to irregular network topologies of our protocol (e.g. see Kirckpatrick 1972 and Hunt 2001 for a review). In addition, a simple statistical approach can be devised for randomly fractured networks. For example, in an all-to-all network where links between cells have been independently removed with probability $q$, the sparseness of the network (i.e. $q$ ) and its size $N$ can be inferred from a sample of measures of connection proximity $k_{1}, \ldots, k_{\mathrm{M}}$, each obtained experimentally in connected pairs. The statistics of distribution of $\{k\}$ can be in fact compared to the probability of observing "proximity 1 ", "proximity 2", ..., "proximity $k$ ", computed as

$$
\begin{aligned}
\operatorname{Pr}\{\text { proximity } 1\}= & (1-q) \\
\operatorname{Pr}\{\text { proximity } 2\}= & (N-2) \cdot(1-q)^{2} \\
& \cdot[1-\operatorname{Pr}\{\text { proximity } 1\}] \\
\operatorname{Pr}\{\text { proximity } 3\}= & (N-2) \cdot(N-3) \cdot(1-q)^{3} \\
& \cdot[1-\operatorname{Pr}\{\text { proximity } 1\}] \\
& \cdot[1-\operatorname{Pr}\{\text { proximity } 2\}] \\
\operatorname{Pr}\{\text { proximity } k\}= & (N-2) \cdot(N-3) \cdot \ldots \cdot(N-k) \\
& \cdot(1-q)^{k} \cdot \prod_{h=1}^{k-1} \times \\
& {[1-\operatorname{Pr}\{\text { proximity } h\}] . }
\end{aligned}
$$

Maximum likelihood estimation techniques (Press et al. 1992; Myung 2003) can then be employed to estimate $q$ and $N$ from the observed statistics of occurrence of $\{k\}$ (Fig. 9). It is interesting to note that the fractured network described above coincides with the Erdõs-Rényi random graph model $G(N, 1-q)$. As expected, $\operatorname{Pr}$ \{proximity k\} shows a phase-transition around $N(1-q) \sim 1$, common to a variety of properties of such a model (Bollobás 2001).

Concluding, we look with great interest to present and future advances in population imaging techniques, involving voltage-sensitive dyes and second-harmonic confocal microscopies (Sacconi et al. 2006). Interestingly, in combination with powerful genetic tools allowing temporally patterned fast photoactivation and photoinhibition of single cells (Zhang et al. 2007), our method might lead to network-level trans-impedance cellular spectroscopy and to non-invasively deciphering microcircuit electrical connectivity in neurons or in glial cells for the first time.

Acknowledgements We are grateful to Drs. M. Hasler, D. Atkinson, I. Segev, A. Zemanian and M. Knaflitz for helpful discussions and to Drs. D. Golomb and D. Ulrich for comments on an earlier version of the manuscript. Support: European Commission "NEURONANO" FP6 grant (NMP4-CT-2006-031847); Sokrates/Erasmus Programme (to C.C.). C.C.'s present address: Dept. of Cell Biology and Morphology, Univ. Lausanne, Switzerland.

\section{References}

Amitai, Y., Gibson, J. R., Beierlein, M., Patrick, S. L., Ho, A. M., Connors, B. W., et al. (2002). The spatial dimensions of electrically coupled networks of interneurons in the neocortex. Journal of Neuroscience, 22(10), 4142-4152.

Atkinson, D., \& van Steenwijk, F. J. (1999). Infinite resistive lattices. The American Journal of Physics, 67, 486-492.

Beierlein, M., Gibson, J. R., \& Connors, B. W. (2000). A network of electrically coupled interneurons drives synchronized inhibition in neocortex. Nature Neuroscience, 3(9), 904-910.

Bennett, M. V. L., \& Zukin, R. S. (2004). Electrical coupling and neuronal synchronization in the mammalian brain. Neuron, 41, 495-511.

Bollobás, B. (2001). Random graphs (2nd ed.). Cambridge: Cambridge University Press.

Brogan, W. L. (1991). Modern control theory. Englewood Cliffs: Prentice Hall.

Carnevale, N. T., \& Hines, M. L. (2006). The NEURON book. Cambridge: Cambridge University Press.

Cossart, R., Petanjek, Z., Dumitriu, D., Hirsch, J., Ben-Ari, Y., Esclapez, M. et al. (2006). Interneurons targeting similar layers receive synaptic inputs with similar kinetics. Hippocampus, 16, 408-420.

Davie, J. T., Kole, M. H., Letzkus, J. J., Rancz, E. A., Spruston, N., Stuart, G. J. et al. (2007). Dendritic patch-clamp recording. Nature Protocols, 1(3), 1235-1247.

Dayan, P., \& Abbott, L. F. (2001). Theoretical neuroscience: Computational and mathematical modeling of neural systems. Cambridge, MA: The MIT Press.

Djurisic, M., Antic, S., Chen, W. R., \& Zecevic, D. (2004). Voltage imaging from dendrites of mitral cells: EPSP attenuation and spike trigger zones. The Journal of Neuroscience, 24(30), 6703-6714. 
Dumitriu, D., Cossart, R., Huang, J., \& Yuste, R. (2007). Correlation between axonal morphologies and synaptic input kinetics of interneurons from mouse visual cortex. Cerebral Cortex, 17(1), $81-91$.

Dupont, E., Hanganu, I. L., Kilb, W., Hirsch, S., \& Luhmann, H. J. (2006). Rapid developmental switch in the mechanisms driving early cortical columnar networks. Nature, 439(7072), 79-83.

Ermentrout, B., Wang, J. W., Flores, J., \& Gelperin, A. (2004). Model for transition from waves to synchrony in the olfactory lobe of limax. Journal of Computational Neuroscience, 17(3), 365-383.

Feynman, R. (1964). Lectures on physics. (Vol 2, Chap 22) Reading, MA: Addison-Wesley.

Galarreta, M., \& Hestrin, S. (2001). Electrical synapses between GABA-releasing interneurons. Nature Reviews Neuroscience, 2 (6), 425-433.

Gao, J., \& Holmes, P. (2006). On the dynamics of electrically-coupled neurons with inhibitory synapses. Journal of Computational Neuroscience, 22(1), 39-61.

Gielen, G., Wambacq, P., \& Sansen, W. M. (1994). Symbolic analysis methods and applications for analog circuits: A tutorial overview. Proceedings of IEEE, 82(2), 287-304.

Hestrin, S., \& Galarreta, M. (2005). Electrical synapses define networks of neocortical GABAergic neurons. Trends in Neurosciences, 28(6), 304-309.

Hormuzdi, S. G., Filippov, M. A., Mitropoulou, G., Monyer, H., \& Bruzzone, R. (2004). Electrical synapses: A dynamic signaling system that shapes the activity of neuronal networks. Biochimica et Biophysica Acta, 1662(1-2), 113-137.

Horowitz, P., \& Hill, W. (1989). The art of electronics (2nd ed.). Cambridge: Cambridge University Press.

Hoshi, H., O'Brien, J., \& Mills, S. L. (2006). A novel fluorescent tracer for visualizing coupled cells in neural circuits of living tissue. The Journal of Histochemistry and Cytochemistry, 54, 1169-1176.

Hsu, S. M., Raine, L., \& Fanger, H. (1981) Use of avidin-biotinperoxidase complex $(\mathrm{ABC})$ in immunoperoxidase techniques: $\mathrm{A}$ comparison between $\mathrm{ABC}$ and unlabeled antibody (PAP) procedures. The Journal of Histochemistry and Cytochemistry, 29(4), 577-580.

Hunt, A. G. (2001). AC hopping conduction: Perspective from percolation theory. Philosophical Magazine B, 81, 875-913.

Hutcheon, B., \& Yarom, Y. (2000). Resonance, oscillation and the intrinsic frequency preferences of neurons. Trends in Neurosciences, 23(5), 216-222.

Kirckpatrick, S. (1972). Percolation and conduction. Reviews of Modern Physics, 45(4), 574-588.

Le Be, J. V., \& Markram, H. (2006). Spontaneous and evoked synaptic rewiring in the neonatal neocortex. Proceedings of the National Academy of Sciences of the United States of America, 103(35), 13214-13219.

Lewis, T., \& Rinzel, J. (2003). Dynamics of spiking neurons connected by both inhibitory and electrical coupling. Journal of Computational Neuroscience, 14(3), 283-309.

Migliore, M., Hines, M. L., \& Shepherd, G. M. (2005). The role of distal dendritic gap junctions in synchronization of mitral cell axonal output. Journal of Computational Neuroscience, 18(2), 151-161.

Myung, I. J. (2003). Tutorial on maximum likelihood estimation. Journal of Mathematical Psychology, 47, 90-100.

Oppenheim, A. V., Schafer, R. W., \& Buck, J. R. (1998). Discrete-time signal processing (2nd edn.). Cambridge: Cambridge University Press.
Press, W., Teukolsky, S. A., Vetterling, W. T., \& Flannery, B. P. (1992). Numerical recipes in C: The art of scientific computing. Cambridge: Cambridge University Press.

Prinz, A. A., \& Fromherz, P. (2003) Effect of neuritic cables on conductance estimates for remote electrical synapses. Journal of Neurophysiology, 89, 2215-2224.

Rall, W. (1969). Time constants and electrotonic length of membrane cylinders and neurons. Biophysical Journal, 9(12), $1483-1508$.

Sacconi, L., Dombeck, D. A., \& Webb, W. W. (2006). Overcoming photodamage in second-harmonic generation microscopy: Realtime optical recording of neuronal action potentials. Proceedings of the National Academy of Sciences of the United States of America, 103(9), 3124-3129.

Salameh, A., \& Dhein, S. (2005). Pharmacology of gap junctions. New pharmacological targets for treatment of arrhythmia, seizure and cancer? Biochimica et Biophysica Acta, 1719(1-2), $36-58$.

Saraga, F., Ng, L., \& Skinner, F. K. (2006). Distal gap junctions and active dendrites can tune network dynamics. Journal of Neurophysiology, 95(3), 1669-1682.

Silberberg, G., \& Markram, H. (2007). Di-synaptic inhibition between pyramidal neurons via martinotti cells in neocortical layer $\mathrm{V}$. Neuron, 53(5), 735-746.

Stuart, G. J., \& Palmer, L. M. (2006). Imaging membrane potential in dendrites and axons of single neurons. Pflügers Archiv, 453(3), 403-410.

Szabadics, J., Lorincz, A., \& Tamás, G. (2001). Beta and gamma frequency synchronization by dendritic GABAergic synapses and gap junctions in a network of cortical interneurons. The Journal of Neuroscience, 21, 5824-5831.

Tamás, G., Buhl, E. H., Lorincz, A., \& Somogyi, P. (2000). Proximally targeted GABAergic synapses and gap junctions in a network of cortical interneurons. Nature of Neuroscience, 3, 366-371.

Traub, R. (1995). Model of synchronized population bursts in electrically coupled interneurons containing active dendritic conductances. Journal of Computational Neuroscience, 2(4), 283-289.

Traub, R. D., Kopell, N., Bibbig, A., Buhl, E. H., LeBeau, F. E. N., \& Whittington, M. A. (2001). Gap junctions between interneuron dendrites can enhance synchrony of gamma oscillations in distributed networks. Journal of Neuroscience, 21, 9476-9486.

Ulrich, D. (2002). Dendritic resonance in rat neocortical pyramidal cells. Journal of Neurophysiology, 87, 2753-2759.

Venezian, G. (1994). On the resistance between two points on a grid. American Journal of Physics, 62(11), 1000-1004.

Wallraff, A., Köhling, R., Heinemann, U., Theis, M., Willecke, K., \& Steinhäuser, C. (2006). The impact of astrocytic gap junctional coupling on potassium buffering in the hippocampus. Journal of Neuroscience, 26(20), 5438-5447.

Weiss, T. F. (1996). Cellular biophysics. (Vol 2) Cambridge, MA: The MIT Press.

Wierzba, G. M., Joshi, V., Srivastava, A., Noren, K. V., \& Svoboda, J. A. (1989). Sspice-Symbolic SPICE for linear active circuits, Proc. of the 32nd Midwest symposium on circuits and systems (pp. 1197-1201). Urbana, IL.

Zhang, F., Wang, L. P., Brauner, M., Liewald, J. F., Kay, K., Watzke, N., et al. (2007) Multimodal fast optical interrogation of neural circuitry. Nature, 446(7136), 633-639. 